\title{
Evaluation of two high-abundance protein depletion kits and optimization of downstream isoelectric focusing
}

\author{
FANGHUA QIU $^{1 *}$, TIEYING HOU ${ }^{2 *}$, DEHONG HUANG ${ }^{3}$, ZHIFENG XUE $^{1}$, \\ DONGYAN LIANG ${ }^{1}$, QIUMING LI ${ }^{1}$ and WEIMIAO LIN ${ }^{1}$ \\ ${ }^{1}$ Department of Clinical Laboratory, Guangzhou Hospital of Traditional Chinese Medicine, Guangzhou, Guangdong 510130; \\ ${ }^{2}$ Department of Laboratory Medicine, Guangdong Academy of Medicine Science and Guangdong General Hospital, \\ Guangzhou, Guangdong 510000; ${ }^{3}$ Department of Neurology, Guangzhou Hospital of \\ Traditional Chinese Medicine, Guangzhou, Guangdong 510130, P.R. China
}

Received November 17, 2014; Accepted August 14, 2015

DOI: $10.3892 / \mathrm{mmr} .2015 .4417$

\begin{abstract}
Disease biomarkers for diagnostic and prognostic purposes are most likely within an extremely low concentration range and are thus masked by the presence of high-abundance proteins. Therefore, removing high-abundance proteins is the main challenge for identifying disease biomarkers. In addition, the solution obtained from high-abundance protein depletion kits contains a rich array of compounds, which interfere with isoelectric focusing (IEF). In the present study, the effect of two commercial kits was evaluated and the downstream IEF protocol was optimized. High-resolution results could be obtained according to the following conditions: The ProteoPrep Blue Albumin and IgG Depletion kit depleted albumin and $\mathrm{IgG}$; immobilized $\mathrm{pH}$ gradient strips (typically $18 \mathrm{~cm}$ ) were rehydrated with sample buffer containing $250 \mu \mathrm{g}$ serum proteins at $30 \mathrm{v}$ for $6 \mathrm{~h}, 60 \mathrm{v}$ for $6 \mathrm{~h}, 200 \mathrm{v}$ for $2 \mathrm{~h}$, $500 \mathrm{v}$ for $2 \mathrm{~h}, 1,000 \mathrm{v}$ for $2 \mathrm{~h}, 5,000 \mathrm{v}$ for $2 \mathrm{~h}, 10,000 \mathrm{v}$ for $2 \mathrm{~h}$ and then focusing at $10,000 \mathrm{v}$ up to $110 \mathrm{k}$ vhs. In addition, the protein spots identified by matrix-assisted laser desorption ionization time-of-flight mass spectrometry demonstrated that all proteins had a low abundance. The present study not only provides a definite and effective method for removing high-abundance proteins, but also provides a proper protocol (protocol C) for downstream IEF. The present study includes a comprehensive investigation of serum proteomics, which paves the way for serum protein research.
\end{abstract}

Correspondence to: Professor Dehong Huang, Department of Neurology, Guangzhou Hospital of Traditional Chinese Medicine, 16 Zhuli Road, Guangzhou, Guangdong 510130, P.R. China

E-mail: dh_huang@163.com

*Contributed equally

Key words: serum, albumin and immunoglobulin G, isoelectric focusing, two-dimensional gel electrophoresis

\section{Introduction}

Proteomics is becoming increasingly important for the development of novel biomarkers of various diseases (1). Serum contains a large variety of proteins (2), which could reflect the health of an individual or the current state of a patient (3). Additionally, the collection of serum is minimally invasive (4). Given this, a rich array of techniques have been used to detect biomarkers of disease in serum.

Disease biomarkers for diagnostic and prognostic purposes are most likely within an extremely low concentration range (5). However, high-abundance proteins in serum, including albumin and immunoglobulin $\mathrm{G}(\mathrm{IgG})$, tend to mask low-abundance proteins. Thus, the depletion of albumin and $\mathrm{IgG}$ is necessary for the detection of biomarkers $(1,6)$.

At present, various techniques and methods have been rapidly developed to deplete albumin and IgG (7). There are three main techniques, including centrifugal ultrafiltration (size-based), immunoaffinity-mediated proteomic separation (antibody-based) and Cibacron Blue and related dye (affinity-based). Centrifugal ultrafiltration is a cheap, convenient method but is not as effective and has a lower reproducibility. Immunoaffinity-mediated proteomic separation has a higher specificity, but is expensive and fails to remove the associated fragments of target proteins. In addition, this technique has a lower sample capacity. Furthermore, it occasionally requires specific techniques, including high-performance liquid chromatography. Dye-ligand affinity chromatography is cheap, has a higher loading capacity and avoids cross-contamination between different samples (8). However, the disadvantage of this technique is a lack of specificity.

Based on the analysis of these methods, in the present study, the Cibacron Blue columns were selected for further assessment. The ProteoPrep Blue Albumin and IgG Depletion kit (PROTBA; Sigma-Aldrich, St. Louis, MO, USA) and the Albumin and IgG Removal kit (GE Healthcare, Waukesha, WI, USA) are two commonly used commercial kits. However, to the best of our knowledge, no study has yet compared the efficiencies of these two kits. In addition, few studies have focused on how to optimize suitable isoelectric focusing (IEF) 
protocols for solutions from the above two kits. IEF is a critical step of two-dimensional electrophoresis (2-DE). The solution obtained from the depletion of highly abundant proteins contains certain materials, including ions, which interfere with downstream IEF. Therefore, the aim of the present study was to evaluate two commercial kits with regards to depletion efficiency, specificity and capacity. In addition, the present study also aimed to optimize the suitable downstream IEF protocol and assess the reproducibility of this approach using the serum of different patients.

\section{Materials and methods}

Materials. Strips, urea, thiourea, dithiothreitol (DTT), immobilized $\mathrm{pH}$ gradient (IPG) buffer, CHAPS, iodoacetamide, glycine, SDS and G250 were all purchased from GE Healthcare. Acetone, methanol, ethanol and acetonitrile were obtained from Sigma-Aldrich. Deionized water was prepared using the Milli-Q system (Millipore Corporation, Bedford, MA, USA) and used for the preparation of all buffers. Ettan DALTsix, Ettan IPGphor 3, Image Scanner II and ImagerMaster software, version 7.0 were purchased from GE Healthcare. The experiments of the current study were approved by the ethics committee of the Guangzhou Hospital of Traditional Chinese Medicine (Guangzhou, China). Written informed consent was obtained from the patients prior to participation.

Serum samples. Serum obtained from two patients with colon cancer (age, 40-64 years; male:female, 1:1) was separated by centrifugation at $3,000 \mathrm{x}$ for $15 \mathrm{~min}$ at $4^{\circ} \mathrm{C}$. The supernatant aliquot was placed into tubes $(500 \mu \mathrm{l}$ per tube) and stored at $-80^{\circ} \mathrm{C}$ until use.

Removal of high-abundance proteins from serum. Albumin and $\mathrm{IgG}$ were depleted according to the manufacturer's instructions. Subsequently, protein quantification was performed using the Quick Start Bradford Protein Assay (Bio-Rad Laboratories, Inc., Hercules, CA, USA) and absorbance was measured at $595 \mathrm{~nm}$ using the Multiskan Spectrum microplate reader (Bio-Rad Laboratories, Inc.), using bovine serum albumin as a protein standard.

\section{SDS-PAGE}

One-dimensional-SDS-PAGE. The depletion efficiency of each approach was evaluated using $10 \%$ polyacrylamide gel. The sample was mixed with 2X sample buffer (GE Healthcare), loaded into each lane and electrophoresed through a Bio-Rad system (Bio-Rad Laboratories, Inc.) using the Laemmli SDS buffering system (25 mM Tris-base, $192 \mathrm{mM}$ glycine and $0.1 \%$ SDS; Bio-Rad Laboratories, Inc.). The gels were stained with Coomassie Blue (GE Healthcare) overnight according to the manufacturer's instructions.

First-dimension separation. Following high-abundance protein depletion, $750 \mu \mathrm{g}$ of protein and rehydration buffer was applied to the $18 \mathrm{~cm}$ ReadyStrip ${ }^{\mathrm{TM}}$ IPG strips (pH 4-7; GE Healthcare). IEF was performed using an IPG III system (GE Healthcare). All the processes above were performed at $20^{\circ} \mathrm{C}$. The focused IEF strips were stored at $-80^{\circ} \mathrm{C}$.
Two-dimensional separation. IPG strips were equilibrated for $15 \mathrm{~min}$ in $50 \mathrm{mM}$ Tris- $\mathrm{HCl}(\mathrm{pH} \mathrm{8.8)}$, containing $6 \mathrm{M}$ urea, $1 \% \mathrm{w} / \mathrm{v}$ SDS, 30\% v/v glycerol and $65 \mathrm{mM}$ DTT (GE Healthcare), and re-equilibrated for $15 \mathrm{~min}$ in the same buffer containing $260 \mathrm{mM}$ iodoacetamide in place of DTT. Two-dimensional gel electrophoresis was performed on 12.5\% SDS-PAGE gel, using the Ettan DALTsix system (GE Healthcare). Two-dimensional gels were run at $1 \mathrm{w}$ per gel for $1 \mathrm{~h}$ at $15^{\circ} \mathrm{C}$ and then $15 \mathrm{w}$ per gel until the tracking dye migrated to within $1 \mathrm{~cm}$ of the bottom of the gel.

Gel staining. The gels were washed in distilled water and stained with colloidal Coomassie or silver (GE Healthcare) according to the manufacturer's instructions.

Image analysis. The 2-DE gel was transferred into distilled water and scanned using the ImageScanner system (GE Healthcare) combined with LabScan software, version 7.0 (GE Healthcare). All gel images were analyzed using ImageMaster 2D Platinum software (GE Healthcare). Gel images from each group were edited and spots were matched. A unique identification number was assigned to matching spots on different gels. Normalization of the spot intensities was conducted according to the total optical density of the gel.

Matrix-assisted laser desorption/ionization mass spectrometry (MALDI-MS) and MS/MS analysis. MALDI-time of flight (TOF)/TOF MS measurements were performed on a Bruker Ultraflex III MALDI-TOF/TOF mass Spectrometer (Bruker Daltonics, Leipzig, Germany) operating in reflectron mode with a $20 \mathrm{kV}$ accelerating voltage and $23 \mathrm{kV}$ reflecting voltage. A saturated solution of $\alpha$-cyano-4-hydroxycinnamic acid in $50 \%$ acetonitrile and $0.1 \%$ trifluoroacetic acid was used as the matrix. The matrix solution $(1 \mu \mathrm{l})$ and sample solution at a ratio of 1:1 was applied onto the Score 384 target well. A standard peptide calibration mix in the mass range 800-3,200 Da (Bruker Daltonics) was analyzed for external calibration of the mass spectrometer. The calibration mix contained: Angiotensin II, angiotensin I, substance P, bombesin, ACTH clip 1-17, ACTH clip 18-39 and somatostatin 28. A series of eight samples were spotted around one external calibration mixture. The SNAP algorithm (S/N threshold: 5; Quality Factor Threshold: 30) in Flex Analysis 3.0 (Bruker Daltonics) was used to identify the 100 most prominent peaks in the mass range m/z 700-4,000. The subsequent MS/MS analysis was performed in a data-dependent manner and the five most abundant ions fulfilling certain preset criteria ( $\mathrm{S} / \mathrm{N}$ higher than 3 and Quality Factor higher than 30) were subjected to high energy collision-induced dissociation analysis. The collision energy was set to $1 \mathrm{keV}$ and nitrogen was used as the collision gas.

Database searching. Peptide mass fingerprints (PMFs) were searched using the program Mascot 2.1 (Matrix Science Ltd., London,UK) against the SwissProt database(version 20091028, 510076 sequences; http://www.uniprot.org/uniprot). The search parameters were as follows: Trypsin digestion with one missed cleavage; carbamidomethyl modification of cysteine as a fixed modification and oxidation of methionine as a variable modification; peptide tolerance maximum, $\pm 0.3 \mathrm{Da}$; 
A

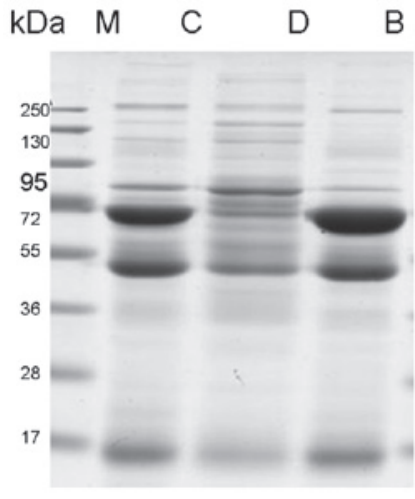

B

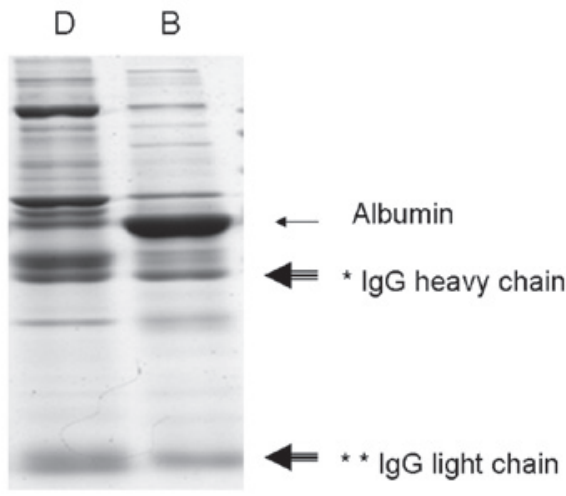

Figure 1. Comparison of the depletion efficiency of two high-abundance protein depletion kits by SDS-PAGE. The serum sample (10 $\mu \mathrm{g}$ ) was loaded onto each lane and separated by $10 \%$ polyacrylamide gels (Coomassie Blue staining). (A) ProteoPrep Blue Albumin and IgG Depletion kit; (B) Albumin and IgG Removal kit. Lanes: M, prestained protein marker; C, crude serum; D, depleted serum; B, bound proteins. Arrows indicate albumin; open arrows indicate protein bands showing IgG *heavy chain and ** light chain.

A

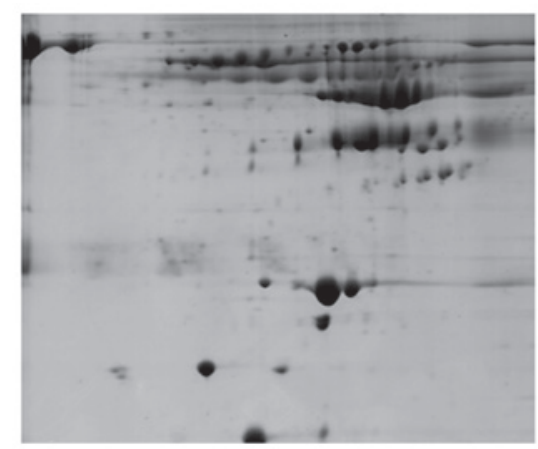

B

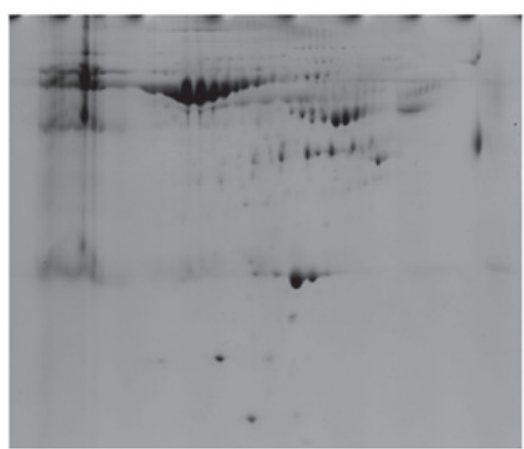

Figure 2. Comparison of the depletion efficiency of two high-abundance protein depletion kits by two-dimensional electrophoresis. The same quantity of proteins (125 $\mu \mathrm{g})$ from the serum samples was loaded onto 2D gel and stained with silver. (A) ProteoPrep Blue Albumin and IgG Depletion kit; (B) Albumin and IgG Removal kit.

MS/MS tolerance maximum, \pm 50 ppm; peptide charge, +1 ; monoisotopic mass. $\mathrm{P}<0.05$ was for a local PMF search. For unambiguous identification of proteins, more than five peptides must be matched for a PMF search.

Different protocols for IEF. The different protocols for IEF were as follows: Protocol A, $1 \mathrm{~h}$ at $200 \mathrm{v}, 2 \mathrm{~h}$ at $10 \mathrm{kv}$ and a final voltage of $8 \mathrm{kv}$ to $80 \mathrm{k}$ vhs; protocol B, $2 \mathrm{~h}$ at $200 \mathrm{v}$ and a final voltage of $8 \mathrm{kv}$ to $80 \mathrm{k}$ vhs; protocol C, $10 \mathrm{~h}$ at $30 \mathrm{v}$ and a final voltage of $10 \mathrm{kv}$ to $100 \mathrm{k}$ vhs; protocol $\mathrm{D}, 3 \mathrm{~h}$ at $200 \mathrm{v}$ and a final voltage of $10 \mathrm{kv}$ to $110 \mathrm{k}$ vhs; protocol $\mathrm{E}$, final voltage of $10 \mathrm{kv}$ to $130 \mathrm{k}$ vhs.

\section{Results}

Depletion efficiency of the kits. The ProteoPrep Blue Albumin and IgG Depletion kit and Albumin and IgG Removal kit are commonly used commercial kits to deplete albumin and IgG. Therefore, in the present study these two kits were compared with regards to depletion efficiency, specificity and capacity.

The present study initially used SDS-PAGE to compare the efficiency of these two kits. As shown in Fig. 1A, albumin and $\mathrm{IgG}$ were almost completely removed by the PROTBA-Sigma kit (Fig. 1A, Lane B). Following depletion, albumin and IgG bound to the column (Fig. 1A, Lane C). By contrast, the Albumin and IgG Removal kit could not effectively deplete albumin and IgG (Fig. 1B, Lane B), and a considerable quantity remained in the serum (Fig. 1B, Lane D). This result suggests that the ProteoPrep Blue Albumin and IgG Depletion kit could effectively deplete albumin and $\operatorname{IgG}$.

The present study aimed to investigate the depletion effect of the above kits. The samples obtained by these two kits were further separated by 2-DE. As shown in Fig. 2, the number of protein spots on the 2D gel obtained from the ProteoPrep Blue Albumin and IgG Depletion kit was higher than that

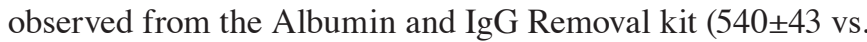
$400 \pm 23$, respectively). The above results demonstrated that the PROTBA-Sigma kit had a higher efficiency compared with the Albumin and $\mathrm{IgG}$ removal kit.

Loading capacity/recovery of protein. The protein yield post-deletion is important for downstream analysis. Therefore, the post-deletion protein yield of these two kits was compared. As shown in Table I, compared with the Albumin and IgG Removal kit, the ProteoPrep Blue Albumin and IgG Depletion kit could load a higher volume (25-50 $\mu \mathrm{l}$ vs. 15-30 $\mu \mathrm{l})$ and produce a higher protein yield (260-540 $\mu \mathrm{g}$ vs. 150-250 $\mu \mathrm{g})$. 
Table I. Approximate yield from two kits.

\begin{tabular}{lccccc}
\hline Kit name & $\begin{array}{c}\text { Loading } \\
\text { volume }(\mu \mathrm{l})\end{array}$ & $\begin{array}{c}\text { Loading } \\
\text { capacity }(\mu \mathrm{g})\end{array}$ & $\begin{array}{c}\text { Recovery } \\
\text { protein }\end{array}$ & Yield (\%) & $\begin{array}{c}\text { Directly apply } \\
\text { to IEF }\end{array}$ \\
\hline ProteoPrep Blue Albumin and & $25-50$ & $1,500-3,000$ & $\begin{array}{l}260 \mu \mathrm{g} / 25 \mu \mathrm{l} \\
540 \mu \mathrm{g} / 50 \mu \mathrm{l}\end{array}$ & $17.33-18.00$ & Yes \\
IgG Depletion kit & $15-30$ & $900-1,800$ & $\begin{array}{l}150 \mu \mathrm{g} / 15 \mu \mathrm{l} \\
250 \mu \mathrm{g} / 30 \mu \mathrm{l}\end{array}$ & $13.66-13.80$ & No \\
Albumin and IgG removal kit & & & & \\
\hline
\end{tabular}

IEF, isoelectric focusing; IgG, immunoglobulin G.

A

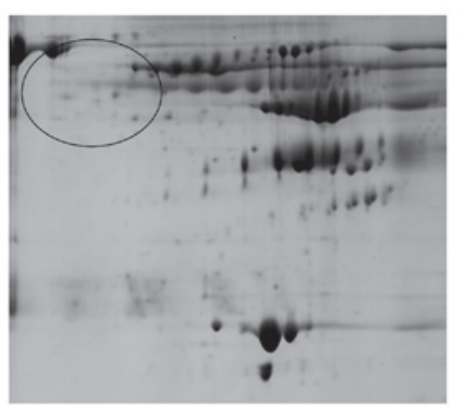

B

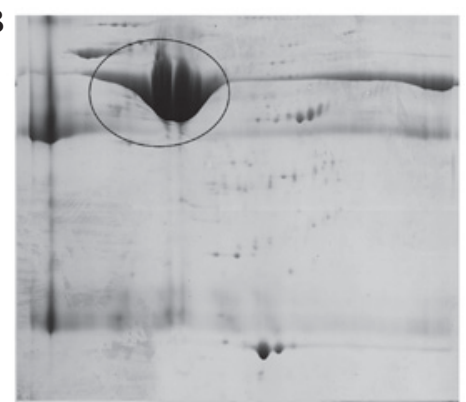

Figure 3. Evaluation of the specificity of bound fractions following depletion using a ProteoPrep Blue Albumin and IgG Depletion kit. The same quantity of proteins $(125 \mu \mathrm{g})$ of the serum samples was loaded onto 2D gel and stained with silver. (A) Serum sample following high-abundance protein depletion. (B) Eluate obtained following depletion of albumin (ellipse). IgG, immunoglobulin G.

Notably, the solution obtained from the ProteoPrep Blue Albumin and IgG Depletion kit can be applied to IEF directly.

Specificity and protein identification. Since the above results demonstrated that the ProteoPrep Blue Albumin and IgG Depletion kit had a higher capacity and higher depletion efficiency, this kit was selected to further assess its specificity.

2D gel was used to analyze samples obtained from the depletion of highly abundant proteins. As shown in Fig. 3, this kit depleted almost all albumin and IgG. By contrast, 2D gel of eluate had more albumin (square) and IgG (ellipse) and less low abundance protein.

Optimization of the IEF protocol. Following the depletion of highly abundant proteins, serum contains additional ions, which may affect downstream IEF. In order to obtain a suitable downstream IEF protocol, five different IEF protocols were assessed as described in Materials and methods. Fig. 4 shows that Protocol A and B led to fewer spots and numerous streaks (Fig. 4A and B). This indicated that proteins form aggregates during IEF. The optimal protocol was $\mathrm{C}$, which resulted in a good resolution of protein spots, less horizontal streaks and the greatest number of spots (Fig. 4C). Protocol D and $\mathrm{E}$ produced more spots but also produced more horizontal streaks (Fig. 4D and E). The number of protein spots on 2-D gels of protocol A, B, C, D and E was 43 $\pm 8,134 \pm 18,538 \pm 45$, $443 \pm 32$ and $404 \pm 33$, respectively.

Subsequently, five unique spots (Fig. 5) from the depleted gels were randomly selected for identification by MALDI-TOF MS. As shown in Table II, the majority of the protein spots, including apolipoprotein E, serotransferrin, heat shock $70 \mathrm{kDa}$ protein 1 , tubulin $\alpha$ chain-like 3 and tropomyosin $\beta$ chain, were predominantly low-abundance proteins.

Assessment of the reproducibility of this approach. To assess the repeatability of our approach, the serum of two patients with colon cancer (age, 40-64; male:female, 1:1) was collected and the optimal protocol was applied. As shown in Fig. 6, the 2D gels exhibited low-abundance proteins and a reduction in abundance, thus demonstrating that the approach had high repeatability.

\section{Discussion}

Given the importance of serum proteomics, this field has progressed rapidly. Albumin and IgG represent $\sim 80 \%$ of the total protein in serum, thus the removal of albumin and IgG is expected to increase the low-abundance protein load by $\sim 4-5$-fold (9). Therefore, removal of albumin and IgG is adequate for the detection of primarily low abundance biomarkers (10). Thus, the depletion of highly abundant proteins is the main challenge of serum proteomics. In addition, the solution obtained from high-abundance protein deletion kits contains a rich array of compounds, which interfere with IEF.

To date, numerous high-abundance protein depletion kits have been designed for the removal of highly abundant proteins from serum (11). Therefore, selecting the most appropriate depletion kit for serum proteomics is important. The selection depends on the balance between the removal of highly abundant proteins and the loss of associated low-abundance proteins. Affinity-based depletion kits are cheaper, have a higher loading capacity and avoid cross-contamination. In 
Table II. Proteins identified from image C, identified by matrix-assisted laser desorption ionization time-of-flight MS.

\begin{tabular}{llrr}
\hline Spot & \multicolumn{1}{c}{ Protein name } & Accession no. & Z-score score \\
\hline 1 & Serotransferrin & P02787 & 61 \\
2 & Heat shock & P01871 & 137 \\
3 & Tubulin alpha chain-like 3 & P02790 & 90 \\
4 & Tropomyosin $\beta$ chain) & P01871 & 87 \\
5 & Apolipoprotein E & P02768 & 136 \\
\hline
\end{tabular}

Spot numbers correspond to those in Fig. 5. The accession number was derived from Swiss-Prot. Scores were calculated using MS-homology software. The score indicates the total of individual peptide scores. The peptide number was detected by MS matched to the identified protein. MS, mass spectrometry.

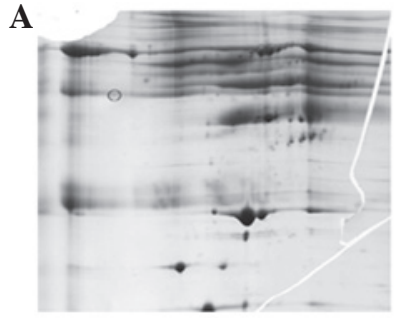

D

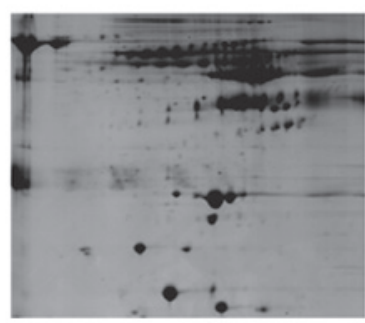

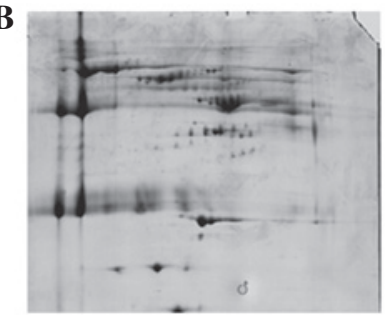

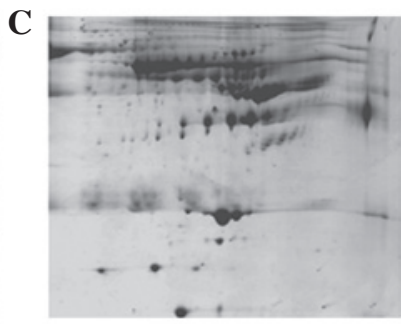

$\mathbf{E}$

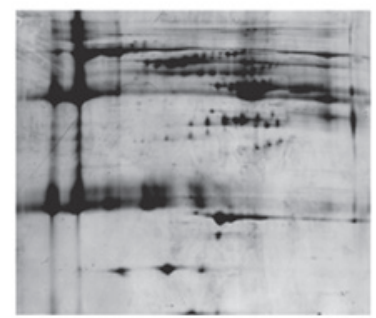

Figure 4. Comparison of different IEF protocols by two-dimensional electrophoresis. Following depletion of high-abundance proteins using the ProteoPrep Blue Albumin and IgG Depletion kit, the same quantity of proteins $(125 \mu \mathrm{g})$ from the serum samples were loaded onto 2D gel and stained with silver. Detailed descriptions of the protocols are described in Materials and methods. The total number of protein spots on 2-D gels of protocol (A), (B), (C), (D) and (E) was $43 \pm 8,134 \pm 18,538 \pm 45,443 \pm 32$ and $404 \pm 33$, respectively.

A

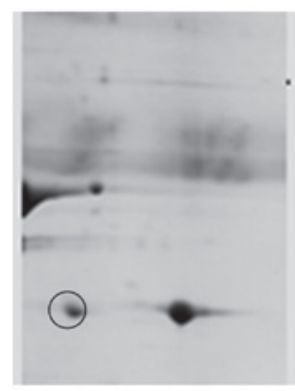

D

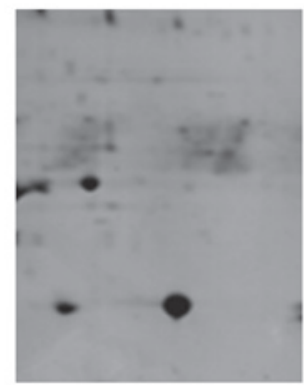

B

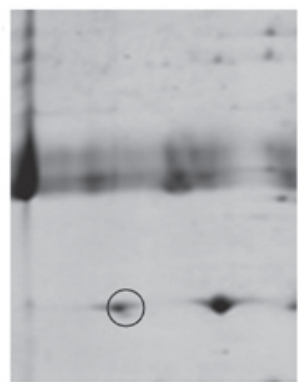

$\mathbf{E}$

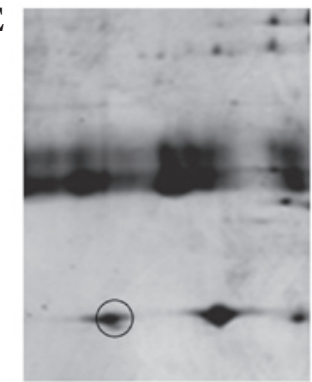

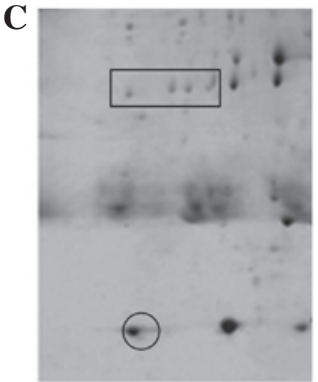

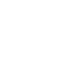



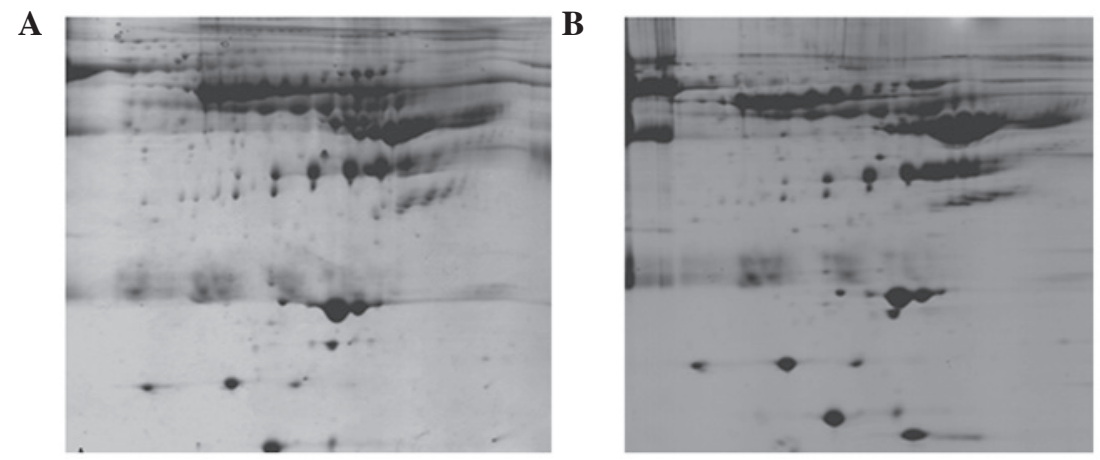

Figure 6. Test reproducibility using the serum of different immune thrombocytopenic purpura patients. The same quantity of proteins $(125 \mu \mathrm{g})$ of the serum samples were loaded onto 2D gel and stained with silver. (A) Patient A; (B) patient B.

addition, this type of kit does not require any specific equipment, thus it is used frequently.

Based on the analysis of all the methods, the Cibacron Blue columns were selected for further investigation. The ProteoPrep Blue Albumin and IgG Depletion kit and the Albumin and IgG Removal kit are based on dye affinity and are commonly used. In the present study, these two kits were compared with regards to depletion efficiency, specificity and capacity. In addition, the protocol for downstream IEF was optimized, which improves the quality of the 2D gel. Finally, test reproducibility of this approach was provided using the serum of different patients.

SDS PAGE and 2D gel clearly demonstrated that the gel which applied the ProteoPrep Blue Albumin and IgG Depletion kit had a higher quality, more protein spots and less albumin. This demonstrated that the ProteoPrep Blue Albumin and IgG Depletion kit could effectively remove high-abundance proteins.

The eluate following albumin and IgG depletion demonstrated that this kit specifically depleted highly abundant proteins. Identification of five unique proteins demonstrated that they were all low-abundance proteins. These results further demonstrated that this kit can specifically deplete high-abundance proteins.

According to a previous study, the protein concentration of the loading sample for silver stained 2-DE gels should not be $<0.5 \mu \mathrm{g} / \mu \mathrm{l}(10)$. Thus, a large quantity of protein used in proteomics studies is often required (12). The present study demonstrated that the ProteoPrep Blue Albumin and IgG Depletion kit can load a higher volume and produce a higher protein yield, which meets the requirement for downstream 2-DE.

In addition, the optimal approach to obtain the most protein is through the simplest procedure. However, the majority of commercial columns require additional steps to remove the interferential materials (13) following depletion of highly abundant proteins, as they use alkaline solution or high level salt solution to elute the unbound protein. They would interfere with IEF. The ProteoPrep Blue Albumin and IgG Depletion kit has notable advantages. For example, the solution obtained following depletion can be used for IEF directly since the wash solution contains fewer ions.

In conclusion, of the two kits, the ProteoPrep Blue Albumin and $\mathrm{IgG}$ Depletion kit was selected for two reasons. Firstly, this kit had high depletion efficiency, specificity and capacity.
Secondly, the solution following depletion could be used for IEF directly.

Normally, during IEF, the electric current must be $<50 \mu \mathrm{A}$, a higher electric current would obstruct IEF. Thus, salt concentration is a critical factor of IEF as a high salt concentration results in a high electric current. Prolonging the low voltage process can remove salts. The present study initially followed standard protocols of IEF [all low voltage steps running for 1 (protocol A) or $2 \mathrm{~h}$ (protocol A and B)]. During IEF, the electric current reached the limitation quickly. $2 \mathrm{D}$ gel produced from protocol $\mathrm{A}$ and B with few protein spots also demonstrated that these two protocols resulted in unsuccessful IEF. Therefore, low voltage steps were prolonged to $3 \mathrm{~h}$ for protocol C, D and E. With this modification, the current decreased to $30 \mu \mathrm{A}$ when the voltage reached $100 \mathrm{kvh}$, indicating that this modification is useful for salt depletion. In addition, three types of final voltage were compared: $100 \mathrm{k}$ vhs (protocol C), $110 \mathrm{k}$ vhs (protocol D) and $130 \mathrm{k}$ vhs (protocol E). 2D gel from protocol C and D exhibited horizontal streaking and fewer protein spots, whereas the $2 \mathrm{D}$ gel of protocol $\mathrm{C}$ resulted in $>500$ protein spots. Overall, protocol C obtains high quality 2D gel with less horizontal streaking and more protein spots, and thus method $\mathrm{C}$ was selected for further investigation.

For high reproducibility, an ideal research approach is required. To confirm the reproducibility of our method, serum was collected from two patients with colon cancer. These two patients received radiotherapy and chemotherapy for $>6$ months. Due to the side effects of the treatments, their serum protein is unique, thus their serum was selected to assess the reproducibility.

Albumin and IgG were depleted using the ProteoPrep Blue Albumin and IgG Depletion kit. Subsequently, the solution obtained underwent IEF with protocol C. Following this approach using serum of patients with colon cancer, high quality 2D gels were obtained, which have low-abundance proteins and reduced high-abundance protein, demonstrating that this approach has high reproducibility.

In conclusion, an effective approach for serum proteomics was successfully provided. This included using the ProteoPrep Blue Albumin and IgG Depletion kit to deplete albumin and $\mathrm{IgG}$, combined with protocol $\mathrm{C}$ to run IEF, which obtains high quality $2 \mathrm{D}$ gels. In addition, five unique proteins were successfully identified by MALDI-TOF/TOF MS and it was demonstrated that they were all low-abundance proteins. The 
above results demonstrated that our approach was highly effective with a high reproducibility, subsequently paving the way for serum proteomics research.

\section{Acknowledgements}

This study was supported by the National Science Foundation of China (grant no. 81053424), the Natural Science Foundation of Guangdong province (grant no. 2014A030313802), the Bureau of Health of Guangzhou Municipality (grant nos. 20141A011016 and 20152A011010), and the Traditional Chinese Medicine Bureau of Guangdong Province (grant no. 2014021).

\section{References}

1. Ahmed N, Barker G, Oliva K, Garfin D, Talmadge K, Georgiou H, Quinn $\mathrm{M}$ and Rice G: An approach to remove albumin for the proteomic analysis of low abundance biomarkers in human serum. Proteomics 3: 1980-1987, 2003.

2. Anderson NL and Anderson NG: The human plasma proteome: History, character, and diagnostic prospects. Mol Cell Proteomics 1: 845-867, 2002.

3. Bjorhall K, Miliotis T and Davidsson P: Comparison of different depletion strategies for improved resolution in proteomic analysis of human serum samples. Proteomics 5: 307-317, 2005.

4. Desrosiers RR, Beaulieu E, Buchanan M and Béliveau R: Proteomic analysis of human plasma proteins by two-dimensional gel electrophoresis and by antibody arrays following depletion of high-abundance proteins. Cell Biochem Biophys 49: 182-195, 2007.
5. Echan LA, Tang HY, Ali-Khan N, Lee K and Speicher DW: Depletion of multiple high-abundance proteins improves protein profiling capacities of human serum and plasma. Proteomics 5: 3292-3303, 2005.

6. Greenough C, Jenkins RE, Kitteringham NR, Pirmohamed M, Park BK and Pennington SR: A method for the rapid depletion of albumin and immunoglobulin from human plasma. Proteomics 4: 3107-3111, 2004.

7. Hoffman SA, Joo WA, Echan LA and Speicher DW: Higher dimensional (Hi-D) separation strategies dramatically improve the potential for cancer biomarker detection in serum and plasma. J Chromatogr B Analyt Technol Biomed Life Sci 849: 43-52, 2007.

8. Kim MR and Kim CW: Human blood plasma preparation for two-dimensional gel electrophoresis. J Chromatogr B Analyt Technol Biomed Life Sci 849: 203-210, 2007.

9. Lescuyer P, Hochstrasser D and Rabilloud T: How shall we use the proteomics toolbox for biomarker discovery? J Proteome Res 6: 3371-3376, 2007.

10. Liu B, Qiu FH, Voss C, Xu Y, Zhao MZ, Wu YX, Nie J and Wang ZL: Evaluation of three high abundance protein depletion kits for umbilical cord serum proteomics. Proteome Sci 9: 24, 2011.

11. Mahn A, Reyes A, Zamorano M, Cifuentes W and Ismail M: Depletion of highly abundant proteins in blood plasma by hydrophobic interaction chromatography for proteomic analysis. J Chromatogr B Analyt Technol Biomed Life Sci 878: 1038-1044, 2010.

12. Qiu F, Huang D, Xiao H, Qiu F, Lu L and Nie J: Detection of tyrosine phosphorylated proteins in hepatocellular carcinoma tissues using a combination of GST-Nck1-SH2 pull-down and two-dimensional electrophoresis. Mol Med Rep 7: 1209-1214, 2013.

13. Thadikkaran L, Siegenthaler MA, Crettaz D, Queloz PA, Schneider P and Tissot JD: Recent advances in blood-related proteomics. Proteomics 5: 3019-3034, 2005. 